\title{
Classical mechanics in reparametrization-invariant formulation and the Schrödinger equation
}

\author{
A. A. Deriglazov and B. F. Rizzut $\mathbb{A}^{\circledast}$ \\ Depto. de Matemática, ICE, Universidade Federal de Juiz de Fora, MG, Brazil and \\ Depto. de Física, ICE, Universidade Federal de Juiz de Fora, MG, Brazil
}

\begin{abstract}
The dynamics of every classical-mechanics system can be formulated in the reparametrizationinvariant (RI) form (that is we use the parametric representation for trajectories, $\mathbf{x}=\mathbf{x}(\tau), t=t(\tau)$ instead of $\mathbf{x}=\mathbf{x}(t))$. In this pedagogical note we discuss what the quantization rules look like for the RI formulation of mechanics. We point out that in this case some of the rules acquire an intuitively clearer form. Hence the formulation could be an alternative starting point for teaching the basic principles of quantum mechanics. The advantages can be resumed as follows. a) In RI formulation both the temporal and the spatial coordinates are subject to quantization. b) The canonical Hamiltonian of RI formulation is proportional to the quantity $\tilde{H}=p_{t}+H$, where $H$ is the Hamiltonian of the initial formulation. Due to the reparametrization invariance, the quantity $\tilde{H}$ vanishes for any solution, $\tilde{H}=0$. So the corresponding quantum-mechanical operator annihilates the wave function, $\hat{\tilde{H}} \Psi=0$, which is precisely the Schrödinger equation, $i \hbar \partial_{t} \Psi=\hat{H} \Psi$. As an illustration, we discuss quantum mechanics of the relativistic particle.
\end{abstract}

\section{INTRODUCTION}

Canonical quantization. The quantum mechanics of a particle can be constructed in several ways. For instance, Feynman discussed the Schrödinger equation from the Dirac integral representation for $\Psi$. [1] Another possible construction is based on physical arguments: the diffraction of electrons, yielding an interference pattern similar to that produced by light suggests the emergence of a wave function governed by a wave equation, which is exactly the Schrödinger equation. [2, 3] We can also apply the canonical quantization procedure [3, 4] to a classical mechanical system with the action

$$
S=\int d t\left[\frac{m}{2}\left(\frac{d \mathbf{x}}{d t}\right)^{2}-V(\mathbf{x}, t)\right],
$$

where $\mathbf{x}=\left(x^{1}, x^{2}, x^{3}\right)$. To achieve this, we rewrite the system in Hamiltonian formalism, in terms of the phasespace variables $x^{i}, p^{i}$ equipped with the Poisson bracket $\left\{x^{i}, p^{j}\right\}=\delta^{i j}$. The basic quantity now is the Hamiltonian $H(\mathbf{x}, \mathbf{p}, t)=\frac{1}{2 m} \mathbf{p}^{2}+V$. According to the canonical quantization paradigm, we associate with the phasespace variables the operators with commutators resembling the Poisson brackets, $\left[\hat{x}^{i}, \hat{p}^{j}\right]=i \hbar \delta^{i j}$,

$$
x^{i} \rightarrow \hat{x}^{i}=x^{i}, \quad p^{i} \rightarrow \hat{p}^{i}=-i \hbar \partial_{i},
$$

and postulate on this base the Schrödinger equation for the wave function $\Psi(t, \mathbf{x})$

$$
i \hbar \frac{\partial}{\partial t} \Psi=\hat{H} \Psi, \quad \hat{H}=H(\hat{\mathbf{x}}, \hat{\mathbf{p}}, t) .
$$

\footnotetext{
*Electronic address: alexei.deriglazov@ufjf.edu.br

${ }^{\dagger}$ Electronic address: brunorizzuti@ufam.edu.br; On leave of absence from Instituto de Saúde e Biotecnologia, ISB, Universidade Federal do Amazonas, AM, Brazil.
}

Reparametrization-invariant formulation. Anyone classical system can be reformulated in the reparametrization-invariant (RI) form. To achieve this, we introduce parametric representation for the trajectory $\mathbf{x}=\mathbf{x}(t)$, say $\mathbf{x}=\mathbf{x}(\tau), t=t(\tau)$, where $\tau$ is an arbitrary parameter along the trajectory. Denoting $\frac{d a}{d \tau} \equiv \dot{a}$, we can write the equalities $d t=\dot{t} d \tau, \frac{d \mathbf{x}}{d t}=\frac{\dot{\mathbf{x}}}{t}$. Using these in Eq. (11) we obtain the action which is invariant under the reparameterizations $\tau \rightarrow \tau^{\prime}=f(\tau)$

$$
\tilde{S}=\int d \tau \tilde{L} ; \quad \tilde{L}=\frac{m \dot{\mathbf{x}}^{2}}{2 \dot{t}}-\dot{t} V(\mathbf{x}, t)
$$

This is equivalent to (11), as the Lagrangian equations for the functions $\mathbf{x}(\tau), t(\tau)$, which follow from (4), imply the correct equations for $\mathbf{x}(t)$. [5]

Comment. We stress that by construction, the parameter $\tau$, as well as the functions $\mathbf{x}(\tau), t(\tau)$ have no direct physical meaning. [6] To illustrate this, let us consider the free particle, $V=0$. Then (4) implies the equations of motion $\left(\frac{\dot{\mathbf{x}}}{t}\right)^{\cdot}=0,\left(\frac{\dot{\mathbf{x}}^{2}}{\dot{t}^{2}}\right)^{\cdot}=0$. Due to the reparametrization invariance, general solution to these equations contains, besides the integration constants $\mathbf{v}$ and $\mathbf{x}_{0}$, an arbitrary function $g(\tau)$

$$
\mathbf{x}=\mathbf{v} g(\tau)+\mathbf{x}_{0}, \quad t=g(\tau) .
$$

These expressions, while determine the straight line (both in $\mathbf{x}$ and in $(t, \mathbf{x})$ spaces), do not specify any definite evolution law along the line. Only the functions $\mathbf{x}(t)$ have the physical meaning. Excluding $\tau$ from the parametric equations (5), we obtain $\mathbf{x}(t)=\mathbf{v} t+\mathbf{x}_{0}$.

Formulation of the problem. It is the aim of this note to reformulate the quantization rules listed above for the RI formulation. Our motivations for this type of presentation of quantum mechanics are as follows.

1. From Eq. (2) there may arise an impression that in quantum mechanics only the spatial coordinates are subject to quantization, see, for example, the text-books in 
Ref. 7 and 8. Moreover, in Ref. 8 it is claimed that it is "surprisingly complicated" to promote time as an operator. As will be shown below, quantization of the temporal coordinate does not represent any special problem in the RI formulation. So, the fact that time is not quantized can be regarded as an artefact of the formulation used, and does not represent an intrinsic property of the quantum-mechanics quantization paradigm.

2. In RI formulation, the quantity that vanishes for any true trajectory of the phase space naturally appears. The quantum counterpart of this quantity is precisely the Schrödinger equation. That is, the RI formulation implies (one more) simple intuitive argument for postulating the Schrödinger equation.

3. Relativistic systems are usually formulated in the RI form, 9 -13] so the familiarity with RI formulation of classical mechanics can be important for proper understanding of the special-relativity theory.

\section{QUANTUM MECHANICS OF THE RI FORMULATION}

The action (44) is defined on configuration space with the coordinates $x^{i}, t$. So, to reach the Hamiltonian formulation, we introduce the phase space parameterized by $x^{i}, p^{i}, t, p_{t}$, with the Poisson brackets defined as

$$
\begin{gathered}
\left\{x^{i}, p^{j}\right\}=\delta^{i j}, \\
\left\{t, p_{t}\right\}=1 .
\end{gathered}
$$

According to the standard Hamiltonization prescription [11, 14], the variables $x^{i}(\tau), t(\tau)$ obey the Euler-Lagrange equations, while the dynamics of the conjugate momenta is specified by the equations

$$
p^{i}=\frac{\partial \tilde{L}}{\partial \dot{x}^{i}}=\frac{m \dot{x}^{i}}{\dot{t}}, p_{t}=\frac{\partial \tilde{L}}{\partial \dot{t}}=-\frac{m \dot{\mathbf{x}}^{2}}{2 \dot{t}^{2}}-V .
$$

These equations imply the constraint 15]

$$
\tilde{H} \equiv p_{t}+\frac{1}{2 m} \mathbf{p}^{2}+V=0,
$$

which is satisfied for any solution to equations of motion. It reappears once again when we try to construct the canonical Hamiltonian of the action (4)

$$
p^{i} \dot{x}^{i}+p_{t} \dot{t}-L=\dot{t}\left[p_{t}+\frac{1}{2 m} \mathbf{p}^{2}+V\right],
$$

which thus vanishes for any true trajectory.

Comment. Being reparametrization invariant, the action (4) represents an example of a theory with local symmetry. The appearance of constraints in the Hamiltonian formalism is a characteristic property of such theories. A systematic method for analysis of a locally-invariant theory has been suggested by Dirac, [16] and is now based on solid mathematical grounds, see, for example, the textbooks in Ref. 9-11. For the exact relationship between local symmetries and Hamiltonian constraints see Ref. 11, 17, 18.

To quantize the RI formulation, we replace the phasespace variables by operators that resemble the brackets (6), (7)

$$
\begin{gathered}
t \rightarrow \hat{t}=t, \quad p_{t} \rightarrow \hat{p}_{t}=-i \hbar \partial_{t}, \\
x^{i} \rightarrow \hat{x}^{i}=x^{i}, \quad p^{i} \rightarrow \hat{p}^{i}=-i \hbar \partial_{i} .
\end{gathered}
$$

Since the phase-space function $\tilde{H}$ vanishes in classical theory, we expect that the corresponding quantummechanical operator annihilates the wave function, $\hat{\tilde{H}} \Psi=0$. Taking into account Eqs. (9), (11), the condition reads

$$
i \hbar \partial_{t} \Psi=\left(-\frac{\hbar^{2}}{2 m} \triangle+V\right) \Psi .
$$

That is, we have arrived at the Schrödinger equation. Comment. In the standard formulation (1) the commutators $\left[\hat{x}^{i}, \hat{p}^{j}\right]=i \hbar \delta^{i j}$, being combined with the formula

$$
(\Delta A)^{2}(\Delta B)^{2} \geq\left(\frac{1}{2 i}[\hat{A}, \hat{B}]\right)^{2},
$$

for the standard deviation of the hermitian operators $\hat{A}, \hat{B}$ (see the derivation on pages 108-109 of Ref. 44), implies $\left(\Delta x^{i}\right)\left(\Delta p^{j}\right) \geq \frac{\hbar}{2} \delta^{i j}$. By this way, the positionmomentum uncertainty relation arises on a fundamental level, as a pure algebraic fact. In contrast, it is well known [4, 20-22] that the energy-time uncertainty relation has very different origin and interpretation. (See also a particular derivation of energy-time uncertainty relation on page 112 of Ref. 4).

In the reparametrization invariant formulation (4) we have, in addition to (6), the commutator $\left\{\hat{t}, \hat{p}_{t}\right\}=i \hbar$. So, one asks whether the energy-time uncertainty relation can be derived in the same way, 23. as an algebraic consequence of (7). We point out, that the appearance of the bracket (7) on an equal footing with (6) does not mean complete symmetrization of the quantum-mechanics formalism with respect to the time and the position variables.

The asymmetry has various origins, some of them are enumerated below: a) Scalar product implies integration on the position variables at a fixed instant. b) The operator $\hat{p}_{t}$ is hermitian only on the subspace of solutions of the Shcrödinger equation, see Eq. (12). c) The canonically conjugated variable for $t$ is $p_{t}$, not $H$.

General case. The recipe also works for the general case. Bearing in mind possible applications for the many-particle systems interacting with an electromagnetic field, let us consider the action

$$
S=\int d t L\left(q^{A}, \frac{d q^{A}}{d t}, t\right)
$$

where $q^{A}=\left(\mathbf{x}_{1}, \mathbf{x}_{2}, \ldots, \mathbf{x}_{n}\right), A=1,2, \ldots, 3 n$ stand for generalized coordinates of $3 n$-dimensional configuration space of $n$ particles. Supposing that the action is 
non-singular, equations for the momenta $p_{A}=\frac{\partial L(q, v, t)}{\partial v^{A}}$ can be resolved algebraically with respect to $v^{A}, v^{A}=$ $v^{A}\left(q^{B}, p^{C}, t\right)$. Then the physical Hamiltonian reads

$$
H\left(q^{A}, p_{A}, t\right)=p_{A} v^{A}-L(q, v, t) .
$$

The RI action reads

$$
\tilde{S}=\int d t \tilde{L}=\int d \tau \dot{t} L\left(q^{A}, \frac{\dot{q}^{A}}{\dot{t}}, t\right) .
$$

This leads to the following equations for conjugate momenta

$$
\begin{gathered}
p^{i}=\left.\frac{\partial L(q, v, t)}{\partial v^{A}}\right|_{v^{A} \rightarrow \frac{\dot{q}^{A}}{t}}, \\
p_{t}=\left.\left[L(q, v, t)-\frac{\dot{q}^{A}}{\dot{t}} \frac{\partial L(q, v, t)}{\partial v^{A}}\right]\right|_{v^{A} \rightarrow \frac{\dot{q}^{A}}{t}} .
\end{gathered}
$$

The equation (17) can be solved as $\frac{\dot{q}^{A}}{\dot{t}}=v^{A}(q, p, t)$. Using this expression in (18), we obtain the constraint $p_{t}+p_{A} v^{A}-L(q, v, t)=0$, or, equivalently

$$
p_{t}+H=0
$$

which is satisfied for any solution to the phase-space equations of motion. Here $H$ stands for the Hamiltonian of the initial formulation $L$. Similarly to the example discussed above, the canonical Hamiltonian of the RI formulation (16) is proportional to the constraint

$$
p_{A} \dot{q}^{A}+p_{t} \dot{t}-\tilde{L}=\dot{t}\left[p_{t}+p_{A} v^{A}-L\right] .
$$

To quantize the RI formulation, we replace the phasespace variables by operators, $p_{t} \rightarrow \hat{p}_{t}=-i \hbar \partial_{t}, p_{A} \rightarrow$ $\hat{p}_{A}=-i \hbar \partial_{A}$, and impose the equation (19) as a constraint on the wave function. This immediately leads to the Schrödinger equation $i \hbar \partial_{t} \Psi=\hat{H} \Psi$.

\section{EXAMPLE: QUANTUM MECHANICS OF THE RELATIVISTIC PARTICLE}

Using the physical coordinates $\mathbf{x}(t)$, the relativistic particle action reads

$$
S=-m c \int d t \sqrt{c^{2}-\left(\frac{d \mathbf{x}}{d t}\right)^{2}}
$$

where $m$ is the mass of the particle and $c$ is the speed of light. Introducing an arbitrary parametrization $\mathbf{x}(\tau)$, $t(\tau)$ of the trajectory, the action acquires the RI form

$$
\tilde{S}=-m c \int d \tau \dot{t} \sqrt{c^{2}-\frac{\dot{\mathbf{x}}^{2}}{\dot{t}^{2}}}
$$

If we agree to consider only the parameterizations adjusted with the "time flow", $\frac{d t}{d \tau}>0$, the action can be written in the manifestly relativistic-invariant form

$$
\begin{aligned}
\tilde{S} & =-m c \int d \tau \frac{\dot{t}}{|\dot{t}|} \sqrt{(c \dot{t})^{2}-\dot{\mathbf{x}}^{2}} \\
& =-m c \int d \tau \sqrt{\eta_{\mu \nu} \dot{x}^{\mu} \dot{x}^{\nu}}
\end{aligned}
$$

where the Minkowsky metric has been chosen as $\eta_{\mu \nu}=$ $(+,-,-,-)$.

Passing to the Hamiltonian formulation for (22), we introduce the canonical momenta

$$
\begin{gathered}
\mathbf{p}=\frac{\partial \tilde{L}}{\partial \dot{\mathbf{x}}}=\frac{m c \dot{\mathbf{x}}}{\dot{t} \sqrt{c^{2}-\frac{\dot{\mathbf{x}}^{2}}{\dot{t}^{2}}}}, \\
p_{t}=\frac{\partial \tilde{L}}{\partial \dot{t}}=-m c \sqrt{c^{2}-\frac{\dot{\mathbf{x}}^{2}}{\dot{t}^{2}}}
\end{gathered}
$$

The first equation can be used to present $\dot{\mathbf{x}}$ through $\mathbf{p}$ and $\dot{t}, \dot{\mathbf{x}}=\dot{t} \frac{c \mathbf{p}}{\sqrt{m^{2} c^{2}+\mathbf{p}^{2}}}$. Using this result in Eq. (25), we obtain the basic constraint $\left.p_{t}=-c \sqrt{m^{2} c^{2}+\mathbf{p}^{2}}\right)$. As it should be, the canonical Hamiltonian turns out to be proportional to the constraint, $\tilde{H}=\dot{t}\left(p_{t}+c \sqrt{m^{2} c^{2}+\mathbf{p}^{2}}\right)$.

Quantizing the model via the RI approach, we arrive at the Shrödinger equation which is just the square-root Klein-Gordon equation [7]

$$
i \hbar \partial_{t} \Psi=c \sqrt{m^{2} c^{2}-\hbar^{2} \triangle} \Psi
$$

where $\triangle=\frac{\partial^{2}}{\partial x_{1}^{2}}+\frac{\partial^{2}}{\partial x_{2}^{2}}+\frac{\partial^{2}}{\partial x_{3}^{2}}$. For the latter use, we write it in the equivalent form

$$
\left[i \frac{\partial}{c \partial t}-\sqrt{\mu^{2}-\triangle}\right] \Psi=0, \quad \mu \equiv \frac{m c}{\hbar} .
$$

It implies the right nonrelativistic limit. Indeed, expanding the square root into power series with respect to $\frac{1}{c^{2}}$ and keeping the leading two terms, we observe that the function $\chi \equiv \exp \left(-i \frac{m c^{2}}{\hbar} t\right) \Psi$ obeys the nonrelativistic Schrödinger equation $i \hbar \partial_{t} \chi=-\frac{\hbar^{2}}{2 m} \triangle \chi$.

Dealing with the equation (27), we are faced with two well-known problems. First, it contains the square-root operator. Second, it has no the manifestly relativisticcovariant form. We demonstrate now that both problems can be avoided, reformulating the theory in the equivalent form in terms of the real scalar field $\phi\left(x^{\mu}\right)$ instead of the complex wave function $\Psi$.

Consider the manifestly relativistic Klein-Gordon equation for the real function $\phi$

$$
\left[\partial_{\mu} \partial^{\mu}+\left(\frac{m c}{\hbar}\right)^{2}\right] \phi=0
$$

The equations (27) and (28) turn out to be equivalent in the following sense: 
A) If $\phi$ is a solution to the Klein-Gordon equation (28), then

$$
\Psi=\Psi_{1}+i \Psi_{2}=-\sqrt{\mu^{2}-\triangle} \phi-i \frac{\partial}{c \partial t} \phi,
$$

obeys the Schrödinger equation (27). We point out an analogy with the electrodynamics: as the vector potential $\mathbf{A}$ produces the electric and magnetic fields, $\mathbf{E}=-\frac{1}{c} \partial_{t} \mathbf{A}$, $\mathbf{B}=\nabla \times \mathbf{A}$, the real field $\phi$ produces the real and imaginary parts of the wave function according to Eq. (29). So, we call $\phi$ the wave-function scalar potential. [19] B) If $\Psi$ is a solution to the Schrödinger equation (27), then the function

$$
\phi=k(\mathbf{x})-c \int_{0}^{t} d \tau \Psi_{2}(\tau, \mathbf{x}) .
$$

obeys the Klein-Gordon equation (28). It has been denoted

$$
k(\mathbf{x})=-\int \frac{d^{3} p}{(2 \pi)^{\frac{3}{2}}} \frac{e^{i \mathbf{p x}} \Psi_{1}(\mathbf{p})}{\sqrt{\mu^{2}+\mathbf{p}^{2}}},
$$

and $\Psi_{1}(\mathbf{p})$ is the Fourier-transformation of $\Psi_{1}(0, \mathbf{x})$, $\Psi_{1}(0, \mathbf{x})=\int \frac{d^{3} p}{(2 \pi)^{\frac{3}{2}}} e^{i \mathbf{p x}} \Psi_{1}(\mathbf{p})$. The function $k(\mathbf{x})$ represents a formal solution to the equation $\sqrt{\mu^{2}-\triangle} k(\mathbf{x})=$ $-\Psi_{1}(0, \mathbf{x})$, the latter is the real part of the equation (27) taken at the instant $t=0$.

According to Eq. (29), the probability density can be presented through the wave-function potential

$$
\Psi^{*} \Psi=\left(\frac{\partial}{c \partial t} \phi\right)^{2}+\left(\sqrt{\mu^{2}-\triangle} \phi\right)^{2}
$$

In turn, it allows us to identify the probability density with the energy density of the field $\phi$. Indeed, the equa- tion of motion (28) can be obtained from the action

$$
S=\int d^{4} x\left[\left(\partial_{0} \phi\right)^{2}-\left(\sqrt{\mu^{2}-\triangle} \phi\right)^{2}\right],
$$

so the right-hand side of Eq. (32) is just the energy density of $\phi$.

In resume, quantum mechanics of the relativistic particle can be reformulated in the manifestly relativisticcovariant form in terms of the real wave-function potential (28). Interaction with the electromagnetic field can be achieved adding the term

$$
S_{\text {int }}=\int d t\left[e A_{0}+\frac{e}{c} A_{i} \frac{d x^{i}}{d t}\right]=\int d \tau \frac{e}{c} A_{\mu} \dot{x}^{\mu}
$$

Repeating the analysis made above, we arrive at the equation (26), where one needs to replace $\partial_{i} \rightarrow \partial_{i}-$ $i \frac{e}{\hbar c} A_{i}, c \partial_{t} \rightarrow \partial_{0}-i \frac{e}{\hbar c} A_{0}$. Unfortunately, in this case we are not able to reformulate the theory in the manifestly relativistic-covariant form.

\section{CONCLUDING REMARKS}

We have shown how the canonical quantization can be reformulated in a fair way: the time variable stands on an equal footing with spatial variables, both being quantized. For that, we work with the reparameterization invariant action, where time and spatial variables are functions of an arbitrary parameter along the trajectory. Reparametrization invariance implies the constraint (19) which holds for any true trajectory. The corresponding quantum-mechanical operator annihilates the wave function, leading precisely to the Schrödinger equation. As an application for the RI formulation, we demonstrated that the Klein-Gordon equation for the real field has the probabilistic interpretation.
[1] David Derbes, "Feynman's derivation of the Schrödinger equation," Am. J. Phys. 64 (7), 881-884 (1996).

[2] Paul Mazur and Robert H. Barron, "On a Variation of a Derivation of the Schrödinger Equation," Am. J. Phys. 42, 600-602 (1974).

[3] L. I. Schiff, Quantum mechanics (McGraw-Hill Book Company, New York, 1949), p. 17. For canonical quantization, see p. 135.

[4] D. J. Griffiths, Introduction to Quantum Mechanics (Prentice Hall, Upper Saddle River, New Jersey, 1995), p. 121.

[5] RI formulation can also be used for geometrization of the potential motion, see A. A. Deriglazov, "Potential motion in a geometric setting: presenting differential geometry methods in a classical mechanics course," Eur. J. Phys. 29, 767-780 (2008).

[6] Of course, some particular parametrizations can admit an interpretation, see, for example, Daniel M. Greenberger, "Theory of Particles with Variable Mass. I. Formalism,"
J. Math. Phys. 11, 2329-2340 (1970) and "Theory of Particles with Variable Mass. II. Some Physical Consequences," J. Math. Phys. 11, 2341-2347 (1970); V. Tapia, Quantum mechanics of time-dependent systems. Construction of pure states, arXiv:quant-ph/9504001 Gerhard Hahne, Phys. Rev. A 80, 062101 (2009).

[7] J. J. Sakurai, Modern Quantum Mechanics, Revised Edition (Addison-Wesley Publishing Company Inc., 1994), p. 68.

[8] M. Srednicki, Quantum Field Theory (Cambridge University Press, 2007), p. 9.

[9] D. M. Gitman and I. V. Tyutin, Quantization of Fields with Constraints (Springer-Verlag, Berlin, 1990).

[10] M. Henneaux and C. Teitelboim, Quantization of Gauge Systems (Princeton Univ. Press, New Jersey, 1992).

[11] A. A. Deriglazov, Classical Mechanics, Hamiltonian and Lagrangian Formalism (Springer-Verlag, Berlin Heidelberg, 2010).

[12] F. A. Berezin and M. S. Marinov, "Classical spin and 
Grassmann algebra," JETP Lett. 21, 320-321 (1975); "Particle Spin Dynamics as the Grassmann Variant of Classical Mechanics," Ann. Phys. 104, 336-362 (1977).

[13] A. A. Deriglazov, A. V. Galajinsky, "Covariant supplementation scheme for infinitely-reducible first-class constraints," Phys. Lett. B 381, 105-112 (1996).

[14] H. Goldstein,Classical Mechanics (Addison-Wesley, Reading, MA, 1980).

[15] The constraint can be written as $p_{t}=-H$, where $H$ is the Hamiltonian of the initial formulation. For this reason, energy is often called the conjugate momentum for the time variable, see C. Lanczos, The variational principles of mechanics (University of Toronto Press, Toronto, $1949)$, pp. 132-133. Observe also that $H(q, p, t)$ is generally not a conserved quantity.

[16] P. A. M. Dirac, "Generalized Hamiltonian dynamics," Can. J. Math. 2, 129-148 (1950); Lectures on Quantum Mechanics (Yeshiva Univ., New York, 1964).

[17] A. A. Deriglazov, K. E. Evdokimov, "Local symmetries in the Hamiltonian framework. 1. Hamiltonian form of the symmetries and the Noether identities," Int. J. Mod.
Phys. A 15, 4045-4067 (2000).

[18] A. A. Deriglazov, "Improved extended Hamiltonian and search for local symmetries," J. Math. Phys. 50, 012907012922 (2009).

[19] The wave-function potential can also be constructed for the nonrelativistic Schrödinger equation with timeindependent potential, see A. A. Deriglazov, "Formal similarity between mathematical structures of electrodynamics and quantum mechanics," Int. J. Theor. Phys. 50, 654-662 (2011).

[20] L. Mandelstam and I.G. Tamm, "The uncertainty relation between energy and time in nonrelativistic quantum mechanics," J. Phys. (USSR) 9, 249-254 (1945).

[21] Y. Aharonov and D. Bohm, "Time in the Quantum Theory and the Uncertainty Relation for Time and Energy," Phys. Rev. 122, 1649-1658 (1961).

[22] P. Busch, "The Time-Energy Uncertainty Relation," Lect. Notes Phys. 734, 73-105 (2008).

[23] We are grateful to the referees for the suggestion to discuss this point. 